\title{
OPTIMAL QUANTUM ERROR CORRECTION FROM HAMILTONIAN MODELS OR PROCESS TOMOGRAPHY*
}

\author{
ROBERT L. KOSUT ${ }^{\dagger}$
}

\begin{abstract}
A method is presented to integrate a complete "black-box" error correction scheme, that takes quantum process tomography as input and iterates until it finds an optimal error correcting encoding and recovery, thus tying theory to experimental decoherence control. The use of tomography in conjunction with optimal error correction has the potential to provide an effective and practical tool for the engineering development of a quantum computer.
\end{abstract}

"In a machine such as this there are very many other problems due to imperfections. ... Until we propose a complete implementation of this, it is very difficult to analyze. At least some of these problems can be remedied in the usual way by techniques such as error correcting codes and so forth, that have been studied in normal computers. But until we find a specific implementation for this computer, I do not know how to proceed to analyze these effects. However, it appears that they would be very important in practice. This computer seems to be very delicate and these imperfections may produce considerable havoc."

- R. P. Feynman [11]

1. Introduction. The current theory of quantum error-correction (QEC) [25, $13,26,20,15,22]$ assumes that a known class of statistical correlation exists between the decoherence processes afflicting different qubits. A major limitation of this approach is that it does not address the decoherence processes that take place in the specific quantum computer implementation. Moreover, in non fault-tolerant QEC one implicitly assumes that the encoding and recovery operations can be implemented via the controls without any error, as if they were uncoupled from the environment. The environment, however, by definition, is always active. As a result, the encoding and/or recovery cannot be separated from their interactions with the environment [2]. In fault-tolerant QEC this problem is addressed by working with certain discrete sets of quantum gates which can be made fault-tolerant via concatenation [3]. While this approach has led to the celebrated threshold theorem of quantum computation [1], the result is extremely costly in terms of qubit resources.

In this paper we develop QEC that corresponds directly to measured errors obtained from estimating a model of the quantum system. This circumvents the need for hypothetical modeling, and is directly applicable to the specific system architecture.

*Dedicated to Brian Anderson on the occasion of his 70th birthday. Research supported by DARPA Grant FA9550-09-1-0710 administered through AFOSR.

†SC Solutions, Inc., 1261 Oakmead Parkway, Sunnyvale, CA 94085. E-mail: kosut@scsolutions.com 
The QEC is iteratively modified by applying it to the system, taking measurements, and optimizing the QEC accordingly. This approach is a simultaneous direct attack on all of the underlying physical decoherence mechanisms, and is in sharp contrast with the "traditional" QEC approach which deals with one source of decoherence at a time.

The iterative procedure combines previous work in optimal QEC and quantum system identification. It is shown in $[23,28,12,17,18]$ that noisy quantum channels which do not satisfy the standard assumptions for perfect correction [25, 13, 26, 20, $15,22]$ can be optimized for the best possible encoding and/or recovery. In these papers, QEC design is cast as a bi-convex optimization problem to maximize channel fidelity, iterating between encoding and recovery, each being a semidefinite program (SDP) [4].

Quantum system identification and/or estimation generally refers to quantum state tomography (QST), quantum process tomography (QPT), or quantum parameter estimation (QPE). For a quantum system whose measurable state is of dimension $n$, QST estimates the $n \times n$ density matrix $\rho$ which is constrained by physical principles to the convex set $\{\rho \geq 0, \operatorname{Tr} \rho=1\}$. In QPT an $n^{2} \times n^{2}$ matrix known as the process matrix is estimated which characterizes the state-to-state dynamics over a fixed time interval. Specifically, under mild conditions the quantum channel which maps an input state $\rho$ to an output state $\hat{\rho}$ can be expressed in a form known as the Kraus operator-sum-representation (OSR) [22]: $\hat{\rho}=\sum_{\mu} S_{\mu} \rho S_{\mu}^{\dagger}$ where the $S_{\mu}$ are the $n \times n$ OSR elements which typically satisfy $\sum_{\mu} S_{\mu}^{\dagger} S_{\mu}=I_{n}$ known as the completion relation which insures that $\operatorname{Tr} \hat{\rho}=1$, hence preserving probability. In QPT each element is expanded in terms of a set of basis matrices for $\mathbf{C}^{n \times n}$, that is, linear combinations of the (usually orthonormal set of) matrices $\left\{B_{i} \in \mathbf{C}^{n \times n}\right\}_{i=1}^{n^{2}}$. This leads to the equivalent input-output form: $\hat{\rho}=\sum_{i, j=1}^{n^{2}} X_{i j} B_{i} \rho B_{j}^{\dagger}$ where $X$ is the process matrix consisting of all the linear coefficients and as a result of the completion relation is constrained to the convex set $\left\{X \geq 0, \sum_{i, j=1}^{n^{2}} X_{i j} B_{i}^{\dagger} \rho B_{j}=I_{n}\right\}$. In both QST and QPT, probability outcomes are linear in the parameters to be estimated. (This is not the case for QPE where the uncertain parameters in a Hamiltonian model are estimated, e.g., [19, 29].)

Since both the quantum state (density matrix) and the process matrix are constrained by the physics to convex sets, approaches to both QPT and QST have naturally gravitated towards the well established methods of least-squares and maximum likelihood, e.g. $[22,8]$. The resulting estimation problem is a convex optimization problem [19], and thus in principle, is tractable [4]. Unfortunately, however, the dimension of the parameter space for QST, and especially for QPT, can be prohibitive: for Hilbert space dimension $n$, QST scales with $n^{2}$ and QPT with $n^{4}$. For $q$-qubits, $n=2^{q}$, and hence scaling for both is exponential in the number of qubits. Although this places a burden on computation, this also places the same burden on resources, 


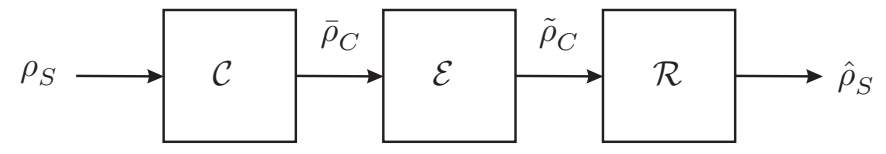

FIG. 2.1. Standard model of error correction system in operator-sum-representation form.

e.g., the number of applied inputs and measurement devices, as well as the number of experiments to achieve a desired accuracy. A number of approaches have been developed to alleviate this scaling burden. Of note are the various forms of ancilla assisted QPT (see [21] for a review), the use of symmetrization to estimate selected process properties [10], and approaches which use prior modeling to simplify process matrix parameters $[5,6]$. With ancilla assistance the scaling power is reduced but is still exponential. Furthermore, ancilla assisted methods may require entangled inputs which are very sensitive to noise and decoherence.

Recently, the use of Compressive Sensing (CS) methods of estimation [9, 7] have been applied to QPT $[24,16]$. CS predicts a scaling of measurement resources of order $s \log N$ where $s$ is the sparsity level of the $N$-dimensional signal to be estimated. In addition, the CS procedure requires solving a convex optimization problem. For QPT with $q$-qubits, $N=\left(2^{q}\right)^{4}$, hence, CS heralds a scaling on the order of $s q$ which is an exponential reduction in resources over standard methods. Recent application with experimental data confirms the simulations [24]. As posited their and in [16], for an initially well designed system whose dynamics are close to a desired unitary (a primary goal in quantum computation) the process matrix, in a basis corresponding to the ideal unitary, is almost sparse, i.e., an $s$-sparse estimate exists which produces an estimation error below any desired level, modulo measurement noise. In other words, the process matrix in this basis is compressible, which makes CS methods feasible. It is clear in quantum information processing that the goal is a desired unitary. In other applications of quantum engineering there may be different criteria, thus requiring selecting a basis for which the estimation is compressible.

Here we will show the efficacy of combining QPT and QEC optimization problems into a single process: iterating between the encoding, the process matrix, and the recovery. Because each step is a convex optimization, the fidelity of encoding and recovery, as well as the fidelity of the process matrix estimate, are all guaranteed to improve through this iteration. Moreover, this approach is particularly appealing because of its potential to directly couple to experiments. An example is presented assuming perfect tomography which illustrates the method and one of the fundamental tradeoff between codespace dimension and number of tomographies.

2. QEC System. The standard model $[25,13,26,20,15,22]$ of an error correction system, shown in the block diagram of Fig. 2.1 is composed of three quantum operations: encoding $\mathcal{C}$, error $\mathcal{E}$, and recovery $\mathcal{R}$. Each of the three operations is 


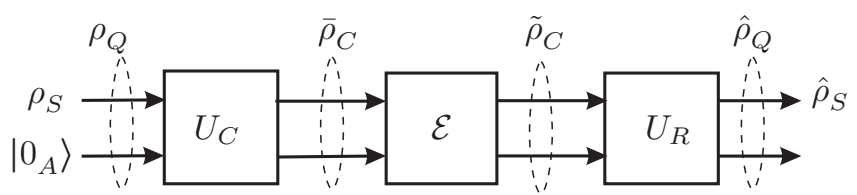

FIG. 2.2. Error correction system showing encoding and recovery unitaries and error system. The input state $\rho_{Q}$, a tensor product of $\rho_{\mathrm{S}}$ the information state and $\left|0_{A}\right\rangle$ the encoding ancilla, is transformed by $U_{C}$ into the codespace. The encoded state is then corrupted by the error channel $\mathcal{E}$ and then recovered by $U_{R}$ to produce the final state $\hat{\rho}_{Q}$.

described as an OSR. Hence, the relation between the input state $\rho_{S}$ and the output state $\hat{\rho}_{S}$ is given by the OSR,

$$
\hat{\rho}_{\mathrm{S}}=\sum_{r, e, c}\left(R_{r} E_{e} C_{c}\right) \rho_{\mathrm{S}}\left(R_{r} E_{e} C_{c}\right)^{\dagger}
$$

The encoding and recovery operation elements are the $n_{C} \times n_{S}$ matrices $\left\{C_{c}\right\}_{c=1}^{m_{C}}$ and the $n_{S} \times n_{C}$ matrices $\left\{R_{r}\right\}_{r=1}^{m_{R}}$. These map between the system Hilbert space (of dimension $n_{S}$ ) and the system + ancillae Hilbert space (of dimension $n_{C}$ ). The error elements are the $n_{C} \times n_{C}$ matrices $\left\{E_{e}\right\}_{e=1}^{m_{E}}$ which represent the effects of decoherence and noise. All the OSRs are assumed to be trace-preserving. Thus,

$$
\sum_{r=1}^{m_{R}} R_{r}^{\dagger} R_{r}=I_{C}, \sum_{e=1}^{m_{E}} E_{e}^{\dagger} E_{e}=I_{C}, \sum_{c=1}^{m_{C}} C_{c}^{\dagger} C_{c}=I_{S}
$$

Any OSR can be implemented as a unitary with ancilla states [22, Thm.8.2] - the Stinespring dilation. Fig. 2.2 depicts one such configuration where the encoding OSR $\mathcal{C}$ is implemented by the $n_{C} \times n_{C}$ encoding unitary, $U_{C}$, which maps the tensor product of the $n_{S} \times n_{S}$ system state $\rho_{\mathrm{S}}$ and the $n_{A} \times 1$ encoding ancilla state $\left|0_{A}\right\rangle$ into the codespace with dimension $n_{C}=n_{S} n_{A}$. Similarly, the recovery OSR is implemented by the $n_{C} \times n_{C}$ recovery unitary, $U_{R}$, operating on the codespace corrupted by the error system $\mathcal{E}$. This model differs from some of the standard models where additional ancilla are used directly in the recovery without passing through the error system. Here we bring all the ancilla together so that the recovery receives a corrupted version of the encoded state.

To relate the encoding and recovery OSR elements to the unitary implementations it is convenient, and without loss of generality, to use the tensor product ordering $\left|0_{A}\right\rangle\left\langle 0_{A}\right| \otimes \rho_{\mathrm{S}}$, and set $\left|0_{A}\right\rangle$ as an $n_{A} \times 1$ vector with a one in the first element and zeros elsewhere. It follows that the $n_{C} \times n_{S}$ encoding matrix $C$ is formed from the first $n_{S}$ columns of $U_{C}$ :

$$
U_{C}=\left[\begin{array}{ll}
C & \tilde{C}
\end{array}\right]
$$

Referring to Fig. 2.2 the noise-free encoded state is given by $\bar{\rho}_{C}=U_{C}\left(\left|0_{A}\right\rangle\left\langle 0_{A}\right| \otimes\right.$ $\left.\rho_{\mathrm{S}}\right) U_{C}^{\dagger}=C \rho_{\mathrm{S}} C^{\dagger}$. This type of encoding is often referred to as a unitary encoding [15] 
because $\mathcal{C}$ has only a single OSR element $\left(m_{C}=1\right)$, the $n_{C} \times n_{S}$ encoding matrix $C$, whose $n_{S}$ columns are the codewords. Because the columns of $C$ are columns of a unitary, they are orthonormal, which is equivalent to satisfying the trace-preserving condition $C^{\dagger} C=I_{S}$. A simple way to make the unitary $U_{C}(2.3)$ from the encoding matrix $C$ is via the SVD of $C$ :

$$
C=U\left[\begin{array}{l}
S \\
0
\end{array}\right] V^{\dagger} \Rightarrow \tilde{C}=\text { last } n_{C}-n_{S} \text { columns of } U
$$

These last columns of $U$ are an orthonormal basis for the nullspace of $C$.

The $n_{S} \times n_{C}$ recovery OSR matrices, $\left\{R_{r}\right\}_{r=1}^{m_{R}}$ form the $n_{C} \times n_{C}$ recovery unitary $U_{R}$ :

$$
U_{R}=\left[\begin{array}{c}
R_{1} \\
\vdots \\
R_{n_{A}}
\end{array}\right]
$$

Observe that because $U_{R}$ is unitary, $m_{R}=n_{A}$. We refer to the matrix $U_{R}$ as the recovery matrix. Because $U_{R}$ is unitary, the trace preserving condition follows: $U_{R}^{\dagger} U_{R}=I_{C}$.

3. Optimal QEC. The error correction objective is to design the encoding $\mathcal{C}$ and recovery $\mathcal{R}$ so that, for a given error system $\mathcal{E}$, the map $\rho_{S} \rightarrow \hat{\rho}_{S}$ is as close as possible to a desired $n_{S} \times n_{S}$ unitary $U_{S}$. A common measure of performance is the average entanglement fidelity between the channel $\mathcal{R E C}$ and the ideal $U_{S}$ :

$$
f=\frac{1}{n_{s}^{2}} \sum_{r, e}\left|\operatorname{Tr} U_{S}^{\dagger} R_{r} E_{e} C\right|^{2}
$$

From [15, 22], $f=1$ if and only if there are constants $\alpha_{r e}$ such that $R_{r} E_{e} C=\alpha_{r e} U_{S}$ with $\sum_{r, e}\left|\alpha_{r e}\right|^{2}=1$. This suggests the indirect measure of fidelity, the "distance-like" error (using the Frobenius norm, $\|X\|_{\text {fro }}^{2}=\operatorname{Tr} X^{\dagger} X$ ),

$$
\begin{aligned}
d & =\sum_{r, e}\left\|R_{r} E_{e} C-\alpha_{r e} U_{S}\right\|_{\text {fro }}^{2} \\
& =\left\|U_{R} E\left(I_{E} \otimes C\right)-\alpha \otimes U_{S}\right\|_{\text {fro }}^{2}
\end{aligned}
$$

with $\alpha$ the $n_{A} \times m_{E}$ matrix with elements $\alpha_{r e}, E$ the $n_{C} \times m_{E} n_{C}$ error system matrix,

$$
E=\left[\begin{array}{lll}
E_{1} & \cdots & E_{m_{E}}
\end{array}\right]
$$

and $U_{R}$ the recovery matrix as defined in(2.5). As shown in [18], (3.1) and (3.2) motivate two optimization problems:

\section{Direct Fidelity Optimization}

$$
\begin{array}{ll}
\text { maximize } & f=\frac{1}{n_{s}^{2}} \sum_{r, e}\left|\operatorname{Tr} U_{S}^{\dagger} R_{r} E_{e} C\right|^{2} \\
\text { subject to } & U_{R}^{\dagger} U_{R}=I_{C}, \quad C^{\dagger} C=I_{S}
\end{array}
$$




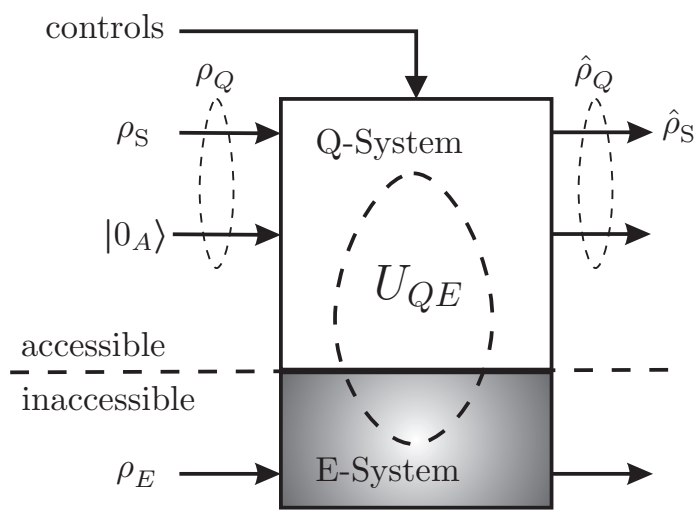

FIG. 4.1. Physical representation of system. The $Q$-system state is accessible: $\rho_{Q}$ and $\hat{\rho}_{Q}$ are states of $Q$ at different times. The E-system state, the environment, is not accessible. The controls may have on effect on both of these systems.

$$
\begin{array}{ll}
\multicolumn{2}{c}{\text { Indirect Fidelity Optimization }} \\
\text { minimize } & d=\left\|U_{R} E\left(I_{E} \otimes C\right)-\alpha \otimes U_{S}\right\|_{\text {fro }}^{2} \\
\text { subject to } & U_{R}^{\dagger} U_{R}=I_{C}, \quad C^{\dagger} C=I_{S}, \quad\|\alpha\|_{\text {fro }}^{2}=1
\end{array}
$$

The relation between fidelity and distance is,

$$
\begin{aligned}
\hat{d} & =\min \left\{d \mid\|\alpha\|_{\text {fro }}=1\right\}=2 n_{S}(1-\sqrt{f}) \\
f & =\left(1-\hat{d} / 2 n_{S}\right)^{2}
\end{aligned}
$$

This establishes that minimizing the distance $d$ is equivalent to maximizing the fidelity $f$.

4. Optimal QEC: iterative OSR modeling . The standard models of error correction such as those shown shown in Fig. 2.1-Fig. 2.2 are not made easily with an OSR obtained directly from a process tomography. Likewise, if the OSR is based on a Hamiltonian model such as depicted in Fig. 4.1. In any case, the standard error models implicitly assume that the encoding and recovery operations (the $Q$ subsystem of $U_{Q E}$ ) can be implemented via the "controls" without any error, as if they were uncoupled from the environment (the $E$-subsystem of $U_{Q E}$ ). The environment, however, by definition, is always active. As a result, the encoding and/or recovery are not readily separated from their interactions with the environment [2]. To see this more clearly, we will compare the two input/output maps from $\rho_{Q} \rightarrow \hat{\rho}_{Q}$ for the system of Fig. 2.2 and Fig. 4.1.

First, for the system of Fig. 2.2,

$$
\hat{\rho}_{Q}=\sum_{e=1}^{m_{E}}\left(U_{R} E_{e} U_{C}\right) \rho_{Q}\left(U_{R} E_{e} U_{C}\right)^{\dagger}
$$

For the system of Fig. 4.1, let the $n_{C} \times n_{C}$ matrices $\left\{A_{e}\right\}_{e=1}^{m_{E}}$ be the OSR of the map 
$\rho_{Q} \rightarrow \hat{\rho}_{Q}$. Thus,

$$
\hat{\rho}_{Q}=\sum_{e=1}^{m_{E}} A_{e} \rho_{Q} A_{e}^{\dagger}, \sum_{e} A_{e}^{\dagger} A_{e}=I_{C}
$$

The $A_{e}$ could be obtained from either a process tomography or from a Hamiltonian model which gives rise to $U_{Q E}$. For a Hamiltonian model representation of Fig. 4.1, assume that $\rho_{E}$ is the pure state $\left|0_{E}\right\rangle$, an $m_{E} \times 1$ vector with a one in the first element and zeros elsewhere. Then the OSR elements of the map $\rho_{Q} \rightarrow \hat{\rho}_{Q}$ are formed from the first $n_{C}$ columns of $U_{Q E}$. Specifically,

$$
U_{Q E}=\left[\begin{array}{cc}
A & \tilde{A}
\end{array}\right], A=\left[\begin{array}{c}
A_{1} \\
\vdots \\
A_{m_{E}}
\end{array}\right]
$$

Since $A$ are the columns of a unitary, the trace preserving condition holds. Equating (4.1) and (4.2) term by term gives the transform pairs,

$$
\left.\begin{array}{l}
A_{e}=U_{R} E_{e} U_{C} \\
E_{e}=U_{R}^{\dagger} A_{e} U_{C}^{\dagger}
\end{array}\right\} e=1, \ldots, m_{E}
$$

This clearly shows the very complicated interactions among the idealized encoding and recovery, $\left(U_{c}, U_{R}\right)$, which are based on an error model with OSR elements $\left\{E_{e}\right\}_{e=1}^{m_{E}}$, and the actual OSR elements $\left\{A_{e}\right\}_{e=1}^{m_{E}}$. The latter, in turn, clearly depend on the encoding and recovery, which in turn depend on the error model, and so on.

Since performance (fidelity or distance) is defined for the map $\rho_{\mathrm{S}} \rightarrow \hat{\rho}_{\mathrm{S}}$, we need also to compare these maps for the two system representations. For the system of Fig. 2.2, the OSR elements are $\left\{R_{r} E_{e} C\right\}_{r, e=1,1}^{n_{A}, m_{E}}$ and the corresponding fidelity is given by (3.1). For the system of Fig. 4.1, the OSR elements are obtained from $U_{Q E}$ using a different arrangement of the columns than that of (4.3), i.e.,

$$
U_{Q E}=\left[\begin{array}{cc}
B & \tilde{B}
\end{array}\right], B=\left[\begin{array}{c}
B_{1} \\
\vdots \\
B_{m_{E} n_{A}}
\end{array}\right]
$$

Thus $\left\{B_{i}\right\}_{i=1}^{m_{E} n_{A}}$ are the $n_{S} \times n_{S}$ OSR elements of the map $\rho_{\mathrm{S}} \rightarrow \hat{\rho}_{\mathrm{S}}$. Observe that these can be determined directly from the OSR elements $\left\{A_{e}\right\}_{e=1}^{m_{E}}$.

The interconnected relations between the two model types motivate an iterative process to balance the two models. Suppose either the $A_{e}$ can be perfectly found from a process tomography or from a Hamiltonian model. The transformation to the $E_{e}$ via (4.4) can be used in an iterative manner to design a new set of encoding and recovery.

Optimal QEC via Iterative OSR Modeling 
Initialize encoding $U_{C}$ and recovery $U_{R}$

Repeat 1-3

\section{OSR Modeling}

(a) Estimate or compute OSR

$$
\left\{A_{e}\right\}_{e=1}^{m_{E}} \rightarrow\left\{B_{i}\right\}_{i=1}^{n_{A} m_{E}}
$$

(b) Compute achieved fidelity

$$
f_{\text {ach }}=\frac{1}{n_{S}{ }^{2}} \sum_{i}\left|\operatorname{Tr} B_{i}\right|^{2}
$$

2. Update error model $\left\{E_{e}=U_{R}^{\dagger} A_{e} U_{C}^{\dagger}\right\}_{e=1}^{m_{E}}$

3. Update QEC

(a) Optimize encoding and recovery

$$
\left\{E_{e}\right\}_{e=1}^{m_{E}} \rightarrow\left(U_{R}, U_{C}\right)
$$

(b) Compute predicted fidelity

$$
f_{\mathrm{p} r e}=\frac{1}{n_{S}{ }^{2}} \sum_{r, e}\left|\operatorname{Tr} U_{S}^{\dagger} R_{r} E_{e} C\right|^{2}
$$

Until $f_{\text {ach }}-f_{\text {pre }}$ is small

This algorithm has several interesting aspects and also highlights some important open problems:

- The fidelity computed in Step $1 \mathrm{~b}, f_{\text {ach }}$, is the achieved fidelity using the OSR from either the Hamiltonian model or from the process tomography. The fidelity in Step $3 \mathrm{~b}, f_{\mathrm{p} r e}$, is the predicted fidelity based on the errors in the form required for the optimal QEC in Step 3. When the achieved and predicted fidelities are close, the algorithm terminates. There is no guaranty at present that this algorithm is convergent, although in all the cases we have run performance improves in a few steps.

- Step 3a requires actually solving two optimization problems. First, finding the optimal encoding and recovery pair $\left(U_{C}, U_{R}\right)$. Secondly, finding a means to implement them via the available controls. Specifically:

- The optimal pair $\left(U_{C}, U_{R}\right)$ is obtained from the bi-convex iterative algorithm described in [18].

- The optimal unitaries provide a target for determining the controls which implement these unitaries. This requires solving a control problem.

- The computational cost using the above iteration consists of the cost to find the optimal encoding and recovery pair in Step 3a, the cost of finding an optimal control to meet these target unitaries, and the cost of obtaining the OSR in Step 1a. The optimal control problem need only be solved in $Q$-space (the codespace) which is considerably smaller than solving for the optimal control over the entire $Q E$-space. The cost to find the OSR depends on whether the procedure is from a Hamiltonian model or from process tomography. In a pure model-based design, the cost is the simulation of the $Q E$-system. In 
a data-based design, the cost of process tomography can be prohibitive. As already mentioned this burden can be greatly relieved using methods from compressive sensing [24].

5. Example: Perfect Tomography. To illustrate the algorithm suppose the $Q E$-system Hamiltonian is,

$$
H_{Q E}(t)=I_{E} \otimes H_{Q}(t)+H_{\mathrm{err}}(t)
$$

where $H_{Q}(t)$ is the time-dependent control Hamiltonian, and $H_{\mathrm{err}}(t)$ is the timedependent error Hamiltonian which includes the environmental dynamics and the coupling to the environment. Assume we have complete control over $H_{Q}(t)$ and set it to the piece-wise constant Hamiltonian,

$$
H_{Q}(t)= \begin{cases}H_{C}=i \log \left(U_{C}\right) & 0 \leq t<1 \\ H_{R}=i \log \left(U_{R}\right) & 1 \leq t \leq 2\end{cases}
$$

where $\left(U_{R}, U_{C}\right)$ are the ideal recovery and encoding operations with $U_{R}=\exp \left(-i H_{R}\right)$ and $U_{C}=\exp \left(-i H_{C}\right)$. Here we have normalized time so that at the end of each unit time interval, the evolution of the control Hamiltonian produces exactly the ideal recovery and encoding unitaries. For purposes of illustration, assume further that the error Hamiltonian is constant and identical over each interval. Then the $Q E$-system unitary, defined at the normalized final time $t=2$, is,

$$
\begin{aligned}
U_{Q E} & =\exp \left(-i H_{R E}\right) \exp \left(-i H_{C E}\right) \\
H_{R E} & =I_{E} \otimes H_{R}+H_{\mathrm{err}} \\
H_{C E} & =I_{E} \otimes H_{C}+H_{\mathrm{err}}
\end{aligned}
$$

For the specific example we want to preserve two qubits $\left(n_{S}=2^{2}=4\right)$ in an environment of fixed dimension, $m_{E}=4$. We set the number of encoding ancilla qubits from $\{0,1,2,3,4\}$. Hence, the number of codespace qubits is $\{2,3,4,5,6\}$, and the Hilbert space of the $Q E$-system is $m_{E} n_{C} \in\{16,32,64,128,256\}$. We run the iterative algorithm 100 times. In each run we let the algorithm go through 10 iterations starting from encoding and recovery unitaries which are identities. In each of the 100 runs we set the $m_{E} n_{C} \times m_{E} n_{C}$ error Hamiltonian $H_{\mathrm{err}}$ randomly and normalized so that $\left\|H_{\mathrm{e} r r}\right\|=0.25$.

Fig. 5.1 shows plots of achieved fidelity vs. the number of codespace qubits. The error bars correspond to 1-standard deviation. The dotted-line is for no iterations (using identity as encoding and recovery), the dot-dashed line is for a single iteration, and the dashed line is for 10 iterations. Each of these iterations requires a tomography. There is clearly a tradeoff between number of codespace qubits and number of tomography iterations. For example, to achieve a fidelity of 0.98 will cost either 1 tomography and 4 codespace qubits, or 10 tomographies and 3 codespace 


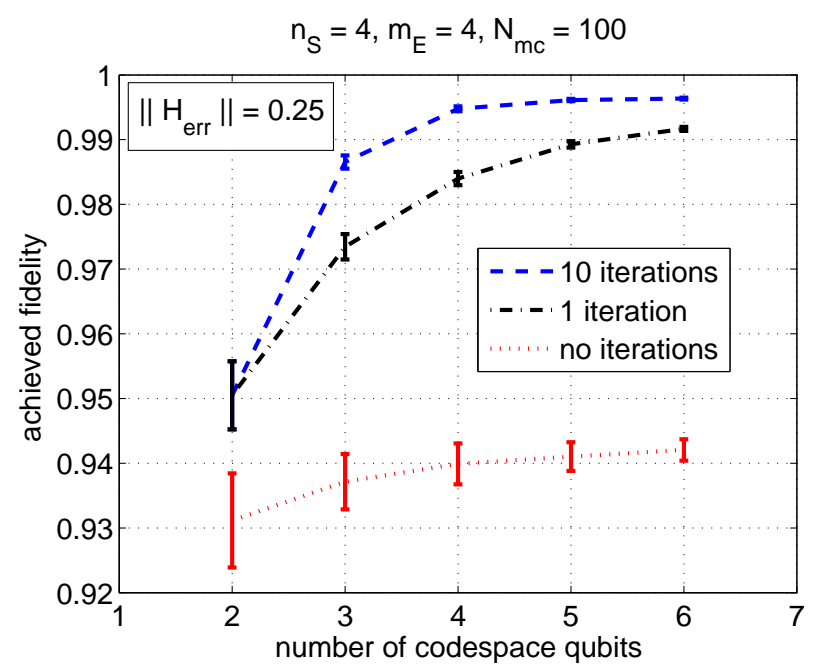

FIG. 5.1. Fidelity vs. number of codespace qubits. The errorbars are 1-standard deviation from 100 runs with $H_{\mathrm{e} r \mathrm{r}}$ chosen randomly for each case shown.

qubits. Similarly, to achieve a fidelity of 0.99 will cost either 1 tomography and 5 codespace qubits, or 10 tomographies and 4 codespace qubits. If tomography can be computationally inexpensive, then minimizing the number of codespace qubits takes priority. A remaining issue, then, is finding a way to do inexpensive quantum system identification.

6. Concluding Remarks. The example illustrating the proposed iterative procedure between QPT and QEC shows an important design tradeoff between codespace size and number of tomographies. There are, however, some caveats. The example does not constrain the control Hamiltonian. Even allowing it to be any arbitrary constant is a stretch in a laboratory setting. However, if the Hamiltonian is, as usual, a linear combination of control variables, it may indeed be possible to achieve the desired unitary in the time allowed. Formulating the problem as a complete optimal control problem in $Q E$-space is, as we mentioned, formidable [14]. Designing the control from process tomography, i.e., from an OSR representation, has not been solved. Learning control, which does require a model of the system, like QPT, requires repeated experimental iterations [27]. Consequently, it is not clear at this time what is the best approach. The example does show that there is an efficacy in separating the control design in $Q$-space from the tomography. Moreover, the advantage of the proposed iteration is that it is data-driven, and hence, amenable to implementation in a laboratory setting. 


\section{REFERENCES}

[1] D. Aharonov, A. Kitaev, and J. Preskill, Fault-tolerant quantum computation with longrange correlated noise, Phys. Rev. Lett., 96 (2006), p. 050504. eprint quant-ph/0510231.

[2] R. Alicki, D.A. Lidar, And P. ZANARdi, Internal consistency of fault-tolerant quantum error correction in light of rigorous derivations of the quantum markovian limit, Phys. Rev. A, $73(2005)$.

[3] P. Aliferis, D. Gottesman, And J. Preskill, Quantum accuracy threshold for concatenated distance-3 codes, Quantum Inf. Comput., 6 (2006), p. 97. eprint quant-ph/0504218.

[4] S. Boyd and L. Vandenberghe, Convex Optimization, Cambridge University Press, Cambridge, UK, 2004.

[5] M. P. A. Branderhorst, I. A. Walmsley, And R. L. Kosut, Quantum process tomography of decoherence in diatomic molecules, in European Conference on Lasers and Electro-Optics, Munich, June 2007.

[6] M. P. A. Branderhorst, I. A. Walmsley, R. L. Kosut, and H. Rabitz, Optimal experiment design for quantum state tomography of a molecular vibrational mode, J. Phys. B: At. Mol. Opt. Phys., 41 (2008).

[7] E. J. Candes, J. Romberg, And T. TaO, Stable signal recovery from incomplete and inaccurate measurements, Comm. Pure Appl. Math., 59 (2006), pp. 1207-1223.

[8] G. M. D'Ariano And P. Lo Presti, Quantum tomography for measuring experimentally the matrix elements of an arbitrary quantum operation, Phys. Rev. Lett., 86 (2001), p. 4195.

[9] D. Donoho, Compressed sensing, IEEE Trans. Inform. Theory, 52 (2006).

[10] J. Emerson, M. Silva, O. Moussa, C. Ryan, M. Laforest, J. Baugh, D. G. Cory, and R. LAflamme, Symmetrised characterisation of noisy quantum processes, Science, 317 (2007), p. 1893.

[11] R. P. Feynman, Quantum mechanical computers, Optics News, 11 (1985), pp. 11-20.

[12] A. S. Fletcher, P. W. Shor, And M. Z. Win, Optimum quantum error recovery using semidefinite programming, Phys. Rev. A, 75 (2007), p. 012338. quant-ph/0606035.

[13] D. GotTesman, Class of quantum error-correcting codes saturating the quantum hamming bound, Phys. Rev. A, 54 (1996), p. 1862.

[14] M. Grace, C. Brif, H. Rabitz, I. Walmsley, R. Kosut, and D. Lidar, Optimal control of quantum gates and suppression of decoherence in a system of interacting two-level particles, J. Phys. B At. Mol. Opt. Phys., 40 (2007), p. S103.

[15] E. Knill and R. Laflamme, Theory of quantum error-correcting codes, Phys. Rev. A, 55 (1997), p. 900.

[16] R. L. Kosut, Quantum process tomography via $\ell_{1}$-norm minimization, arXiv:0812.4323v1[quant-ph], (2008).

[17] R. L. Kosut And D. A. LidaR, Quantum error correction via convex optimization, Springer: Quantum Information Processing, 8 (2009), pp. 443-459. DOI: 10.1007/s11128-009-0120-2.

[18] R. L. Kosut, A. Shabani, And D. A. Lidar, Robust quantum error correction via convex optimization, Phys. Rev. Lett., 100 (2008), p. 020502. (arXiv:quant-ph/0703274).

[19] R. L. Kosut, I. A. Walmsley, And H. Rabitz, Optimal experiment design for quantum state and process tomography and hamiltonian parameter estimation, quant-ph/0411093, (2004).

[20] R. Laflamme, C. Miquel, J.P. Paz, and W.H. Zurek, Perfect Quantum Error Correction Code, Phys. Rev. Lett., 77 (1996), p. 198. eprint quant-ph/9602019.

[21] M. Mohseni, A. T. Rezakhani, and D. A. Lidar, Quantum process tomography: Resource analysis of different strategies, Phys. Rev. A, 77 (2008), p. 032322.

[22] M.A. Nielsen And I.L. ChuAng, Quantum Computation and Quantum Information, Cambridge University Press, Cambridge, UK, 2000.

[23] M. Reimpell AND R. F. Werner, Iterative optimization of quantum error correcting codes, 
Phys. Rev. Lett., 94 (2005), p. 080501.

[24] A. Shabani, R. L. Kosut, M. Mohseni, H. Rabitz, A. Fedrizzi, M. P. Almeida, M. A. Broome, AND A. G. White, Efficient measurement of quantum dynamics via compressive sensing, arXiv:0910.5498[quant-ph], Physical Review Letters (March 11, 2011).

[25] P.W. Shor, Scheme for reducing decoherence in quantum memory, Phys. Rev. A, 52 (1995), p. R2493.

[26] A. M. Steane, Error correcting codes in quantum theory, Phys. Rev. Lett., 77 (1996), p. 793.

[27] I. Walmsley and H. Rabitz, Quantum physics under control, Physics Today, 56 (2003), pp. 43-49.

[28] N. Yamamoto, S. Hara, and K. Tsumara, Suboptimal quantum error correcting procedure based on semidefinite programming, Phys. Rev. A, 71 (2005), p. 022322.

[29] K. C. Young, M. Sarovar, R. L. Kosut, and K. B. Whaley, Optimal quantum multiparameter estimation as applied to dipole- and exchange-coupled qubits, Physical Review A, 79 (2009), p. 062301. 